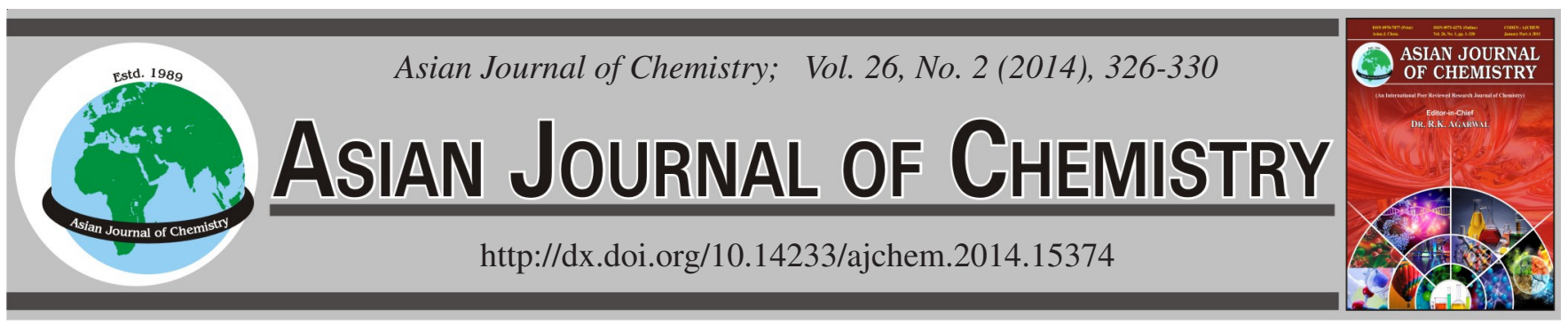

\title{
Synthesis, Spectral Characterization and $\alpha$-Chymotrypsin Activity of 7-O-Substituted Derivatives of 7-Hydroxy-4-methyl-1-benzopyran-2-one
}

\author{
Aziz-ur-Rehman ${ }^{1, *}$, Tehseen Ilyas ${ }^{1}$, Muhammad Athar Abbasi ${ }^{1}$, Khadija Nafeesa $^{1}$, \\ Shaista KhaliQ ${ }^{1}$, Kaniz Rubab ${ }^{1}$, Ghulam Hussain $^{1}$, ShaZia Khurshid ${ }^{1}$ and Irshad Ahmad ${ }^{2}$
}

${ }^{1}$ Department of Chemistry, Government College University, Lahore-54000, Pakistan
${ }^{2}$ Department of Pharmacy, The Islamia University of Bahawalpur, Bahawalpur-63100, Pakistan

*Corresponding author: Tel: +92 42 111000010, Ext. 450; E-mail: azizryk@yahoo.com

\begin{abstract}
In this work, a series of $O$-alkyl/arenyl/acyl substituted derivatives of 7-hydorxy-4-methyl-1-benzenpyran-2-one (3a-l) was synthesized. The parent compound 7-hydroxy-4-methyl-1-benzenpyran-2-one (1) was geared up by the coupling of resorcinol (a) with ethylacetoacetate (b) in the presence of conc. sulphuric acid. Further, $O$-substituted derivatives of parent compound were prepared by treating with different electrophiles (2a-l) using sodium hydride as base and DMF as a solvent. The structure of these synthesized compounds were characterized by IR, EI-MS and ${ }^{1} \mathrm{H}$ NMR. These derivatives were also screened against $\alpha$-chymotrypsin enzyme to check their enzyme inhibition activity. All the compounds displayed $\alpha$-chymotrypsin activity to varying degree.
\end{abstract}

Keywords: 7-Hydroxy-4-methyl-1-benzopyran-2-one, $\alpha$-Chymotrypsin enzyme, ${ }^{1}$ H NMR, EI-MS.

\section{INTRODUCTION}

The coumarin present in nature is a significant member of benzopyrones ${ }^{1}$. Coumarin was naturally extracted from tonka beans ${ }^{2}$ and it is an important constituent of cinnamon and dates ${ }^{3-5}$. Coumarin has the properties of anticoagulant, insecticide, antibacterial and pharmacological functions ${ }^{6}$. Natural and synthesized coumarin is one of the main human metabolite and in human diet, it acts as dietary antioxidant. It also reduces the blood glucose level in human body ${ }^{7}$. Organic and medicinal chemists have considerable attraction for coumarin and its derivatives because of its antiinflammatory/ anti-oxidant activities ${ }^{8}$. In bakery and beverage industry, it is used as flavoring agents and enhanced the flavors of different bakery items 9 . Efforts have been made for the extraction of naturally occurring coumarin from numerous plants and artificial synthesis of coumarin compounds, so that it can be further utilized as the potential drug in medicinal field ${ }^{10}$.

Among the enzymes involved in extracellular matrix degradation, certain serine proteases (elastase, collagenase, cathepsin $\mathrm{G}$ and chymotrypsin) are able to solubilize fibrous proteins such as elastin and collagen ${ }^{11}$. Potent inhibitors have the potential to be developed as new therapeutic agents. In vertebrates, serine protease inhibitors have been studied for many years and they are known to be involved in phagocytosis, coagulation, complement activation, fibrinolysis and blood pressure regulation. In the last decade, it became obvious that in invertebrates, serine proteases and their inhibitors are also involved in parallel physiological processes, for example the blood clotting cascade in Limulus ${ }^{12}$ and the innate immune response $^{13}$. Therefore, the discovery of potent and safe inhibitors has been a very important area of pharmaceutical research. Coumarin and its derivatives have attracted organic chemists because of its remarkable role in biological activities.

The present work is a successful effort to synthesize such compounds exhibiting diverse and improved pharmacological potential. We have synthesized the $O$-alkyl/arenyl/acyl substituted derivatives of 7-hydorxy-4-methyl-1-benzenpyran-2-one with an objective to search new contenders of drug having significant enhanced activity and could be helpful in controlling many degenerative diseases.

\section{EXPERIMENTAL}

All melting points are in degree centigrade and were recorded on Griffin and George apparatus by using open capillary tube. Thin layer chromatography technique was performed on pre-coated silica gel G-25-UV $\mathrm{UV}_{254}$ plates using mixture of $n$-hexane and ethyl acetate as solvents in different ratios for each reaction to check the purity of synthesized compound by giving single spot of product. UV light of 254 $\mathrm{nm}$ wavelength and ceric sulphate reagent were used for this detection. IR technique was performed on instrument Jasco- 
320-A spectrophotometer (wave number in $\mathrm{cm}^{-1}$ ) using $\mathrm{KBr}$ pellets. Numbers of protons calculated by nuclear magnetic resonance spectra also helped in identification of structures and were recorded on a Bruker spectrometer operating at 300 $\mathrm{MHz}$ in $\mathrm{CD}_{3} \mathrm{OD}$ solution. Chemical shifts are quoted in ppm. Mass spectra (EIMS) were given on JMS-HX-110 spectrometer. Resorcinol, ethylacetoacetate and the other electrophiles (methyl iodide, ethyl iodide, isopropyl iodide, allyl iodide, 2-phenylethyliodide, 2-chlorobenzylchloride, 4-bromobenzylbromide, 4-flurobenzyl chloride and 4-chlorobenzyl chloride) were purchased from Sigma Aldrich and Alfa Aesar through local suppliers. All the other employed solvents were of analytical grade.

Procedure for the synthesis of 7-hydroxy-4-methyl-1benzopyran-2-one (1): Resorcinol (4.4 g; a) and ethylacetoacetate $(5 \mathrm{~mL}$; b) was taken in iodine flask. Reaction mixture was shaken well until reaction mass become clear by keeping the flask on water bath and temperature was maintained at $40{ }^{\circ} \mathrm{C}$. Then, cooled concentrated sulphuric acid $(15 \mathrm{~mL})$ was added to the iodine flask and the reaction mixture was placed for overnight. Next day, the chilled water was added in the solution with continuous stirring. The reaction mixture was reserved at room temperature for $0.5 \mathrm{~h}$; dark yellow solid was filtered and washed with distilled water to afford 7-hydroxy4-methyl-1-benzopyran-2-one (1) on drying.

General procedure for the synthesis of $\boldsymbol{O}$-substituted derivatives 2a-i: Compound (1) (2.80 mmol, $0.5 \mathrm{~g})$ was taken in round bottom flask and $\mathrm{N}, \mathrm{N}$-dimethyl formamide (around $5 \mathrm{~mL}$ ) and sodium hydride $(2.80 \mathrm{mmol} ; 0.01 \mathrm{~g}$, ) were added in it at room temperature. The reaction mixture was stirred for $15 \mathrm{~min}$ and then the electrophiles $(2.80 \mathrm{mmol}$; $\mathbf{2 a - i})$ were added into the mixture. The reaction mass was further stirred and monitoring through TLC. After completion of the reaction, the reaction mixture was quenched with cold water $(150 \mathrm{~mL})$. The received solid was filtered, washed with water and dried to yield the corresponding $O$-substituted derivatives of 7-hydroxy4-methyl-1-benzopyran-2-one (3a-i).

General procedure for the synthesis of compounds $3 \mathbf{j}-\mathbf{l}$ : Compound 1 ( $2.8 \mathrm{mmol} ; 0.5 \mathrm{~g}$ ) and $20 \mathrm{~mL}$ of $5 \%$ sodium hydroxide solution was taken in the round bottom flask. The mixture was stirred till solution become clear and then the corresponding electrophiles $(2.80 \mathrm{mmol} ; \mathbf{2} \mathbf{j}$-l $)$ were added into the reaction mixture with constant stirring at room temperature until precipitates were formed. Precipitates were filtered, washed with distilled water and dried to yield the corresponding derivatives (3j-1).

\section{Characterization of the synthesized compounds}

7-Hydroxy-4-methyl-1-benzopyran-2-one (1): Dark yellow amorphous powder; yield $92 \%$; m.p. $188-190^{\circ} \mathrm{C}$; m.f.: $\mathrm{C}_{10} \mathrm{H}_{8} \mathrm{O}_{3}$; m.w. 176; IR ( $\left.\mathrm{KBr}, \nu_{\max }, \mathrm{cm}^{-1}\right)$ : 3550 (O-H stretching), 3418 (C-H aromatic stretching), 1717 ( $\mathrm{C}=\mathrm{O}$ stretching), 1629 $\left(\mathrm{C}=\mathrm{C}\right.$ stretching of aromatic ring); ${ }^{1} \mathrm{H} \mathrm{NMR}\left(300 \mathrm{MHz}, \mathrm{CD}_{3} \mathrm{OD}\right.$ $\delta / \mathrm{ppm}): 7.58$ (d, $J=8.7 \mathrm{~Hz}, 1 \mathrm{H}, \mathrm{H}-5), 6.82$ (dd, $J=8.7,2.4$ Hz, 1H, H-6), 6.70 (d, J =2.4 Hz,1H, H-8), 6.09 (s, 1H, H-3), $2.41\left(\mathrm{~d}, J=0.9 \mathrm{~Hz}, 3 \mathrm{H}, \mathrm{CH}_{3}-4\right)$. EI-MS: $m / z, 176[\mathrm{M}]^{+}, 159$ $\left[\mathrm{C}_{10} \mathrm{H}_{7} \mathrm{O}_{2}\right]^{+}, 144\left[\mathrm{C}_{9} \mathrm{H}_{4} \mathrm{O}_{2}\right]^{+}, 108\left[\mathrm{C}_{6} \mathrm{H}_{4} \mathrm{O}_{2}\right]^{+}, 51\left[\mathrm{C}_{4} \mathrm{H}_{3}\right]^{+}$.

7-Methoxy-4-methyl-1-benzopyran-2-one (3a): White amorphous powder; yield $58 \%$; m.p. $88-90{ }^{\circ} \mathrm{C}$; m.f.: $\mathrm{C}_{11} \mathrm{H}_{10} \mathrm{O}_{3}$; m.w. 190; IR ( $\left.\mathrm{KBr}, v_{\max }, \mathrm{cm}^{-1}\right)$ : 3535 (O-H stretching), 3401 (C-H aromatic stretching), 1729 ( $\mathrm{C}=\mathrm{O}$ stretching), $1616(\mathrm{C}=\mathrm{C}$ stretching of aromatic ring). ${ }^{1} \mathrm{H} \mathrm{NMR}\left(300 \mathrm{MHz}, \mathrm{CD}_{3} \mathrm{OD} \delta /\right.$ ppm): 7.60 (d, $J=8.4 \mathrm{~Hz}, 1 \mathrm{H}, \mathrm{H}-5), 6.80$ (dd, $J=8.7,2.5 \mathrm{~Hz}$, $1 \mathrm{H}, \mathrm{H}-6), 6.69$ (d, J =2.5 Hz, 1H, H-8), 6.09 (s, 1H, H-3), 3.78 (s, 3H, $\left.\mathrm{OCH}_{3}-1\right), 2.41$ (s, 3H, $\left.\mathrm{CH}_{3}-4\right)$. EI-MS: m/z 190 $[\mathrm{M}]^{+}, 175\left[\mathrm{C}_{10} \mathrm{H}_{7} \mathrm{O}_{3}\right]^{+}, 159\left[\mathrm{C}_{10} \mathrm{H}_{7} \mathrm{O}_{2}\right]^{+}, 144\left[\mathrm{C}_{9} \mathrm{H}_{4} \mathrm{O}_{2}\right]^{+}, 108$ $\left[\mathrm{C}_{6} \mathrm{H}_{4} \mathrm{O}_{2}\right]^{+}, 51\left[\mathrm{C}_{4} \mathrm{H}_{3}\right]^{+}$.

7-Ethoxy-4-methyl-1-benzopyran-2-one (3b): Buff amorphous powder; yield $76 \%$; m.p. $98-100{ }^{\circ} \mathrm{C}$; m.f.: $\mathrm{C}_{12} \mathrm{H}_{12} \mathrm{O}_{3}$; m.w. 204; IR (KBr, $v_{\max }, \mathrm{cm}^{-1}$ ): 3539 (O-H stretching), 3407 (C-H aromatic stretching), 1727 ( $\mathrm{C}=\mathrm{O}$ stretching), $1614(\mathrm{C}=\mathrm{C}$ stretching of aromatic ring). ${ }^{1} \mathrm{H}$ NMR $\left(300 \mathrm{MHz}, \mathrm{CD}_{3} \mathrm{OD} \delta /\right.$ ppm): 7.68 (d, $J=8.7 \mathrm{~Hz}, 1 \mathrm{H}, \mathrm{H}-5), 6.95$ (dd, $J=8.7,2.4 \mathrm{~Hz}$, 1H, H-6), 6.87 (d, J =2.4 Hz, 1H, H-8), 6.15 (s, 1H, H-3), 4.11 (q, $\left.J=6.9 \mathrm{~Hz}, 2 \mathrm{H}, \mathrm{CH}_{2}-1\right), 2.43$ (s, 3H, $\left.\mathrm{CH}_{3}-4\right), 1.42$ (t,

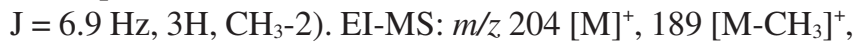
$175\left[\mathrm{C}_{10} \mathrm{H}_{7} \mathrm{O}_{3}\right]^{+}, 159\left[\mathrm{C}_{10} \mathrm{H}_{7} \mathrm{O}_{2}\right]^{+}, 144\left[\mathrm{C}_{9} \mathrm{H}_{4} \mathrm{O}_{2}\right]^{+}, 108\left[\mathrm{C}_{6} \mathrm{H}_{4} \mathrm{O}_{2}\right]^{+}$, $51\left[\mathrm{C}_{4} \mathrm{H}_{3}\right]^{+}$.

7-Isopropoxy-4-methyl-1-benzopyran-2-one (3c): Offwhite amorphous powder; yield $78 \%$; m.p: 88-90 ${ }^{\circ} \mathrm{C}$; m.f.: $\mathrm{C}_{13} \mathrm{H}_{14} \mathrm{O}_{3}$; m.w. 218; IR (KBr, $v_{\max }, \mathrm{cm}^{-1}$ ): 3542 (O-H stretching), 3406 (C-H aromatic stretching), $1728 \mathrm{~cm}^{-1}$ (C=O stretching), $1613(\mathrm{C}=\mathrm{C}$ stretching of aromatic ring $) .{ }^{1} \mathrm{H}-\mathrm{NMR}(300 \mathrm{MHz}$, $\left.\mathrm{CD}_{3} \mathrm{OD} \delta / \mathrm{ppm}\right): 7.67$ (d, $\left.J=8.7 \mathrm{~Hz}, 1 \mathrm{H}, \mathrm{H}-5\right), 6.92$ (dd, $J=$ 8.7, $2.4 \mathrm{~Hz}, 1 \mathrm{H}, \mathrm{H}-6), 6.86$ (d, $J=2.4 \mathrm{~Hz}, 1 \mathrm{H}, \mathrm{H}-8), 6.13$ (s, $1 \mathrm{H}, \mathrm{H}-3), 4.72$ (m, 1H, H-1), 2.43 (s, 3H, $\left.\mathrm{CH}_{3}-4\right), 1.34$ (d, J= $6.0 \mathrm{~Hz}, 6 \mathrm{H}, \mathrm{H}-2$ \& 3). EI-MS: $m / z, 218[\mathrm{M}]^{+}, 175\left[\mathrm{C}_{10} \mathrm{H}_{7} \mathrm{O}_{3}\right]^{+}$, $159\left[\mathrm{C}_{10} \mathrm{H}_{7} \mathrm{O}_{2}\right]^{+}, 144\left[\mathrm{C}_{9} \mathrm{H}_{4} \mathrm{O}_{2}\right]^{+}, 108\left[\mathrm{C}_{6} \mathrm{H}_{4} \mathrm{O}_{2}\right]^{+}, 51\left[\mathrm{C}_{4} \mathrm{H}_{3}\right]^{+}$.

7-Allyoxy-4-methyl-1-benzopyran-2-one (3d): Creamy white amorphous powder; yield $78 \%$; m.p. $154-157^{\circ} \mathrm{C}$; m.f.: $\mathrm{C}_{13} \mathrm{H}_{12} \mathrm{O}_{3}$; m.w. 216; IR (KBr, $v_{\max }, \mathrm{cm}^{-1}$ ): 3503 (O-H stretching), 3410 (C-H aromatic stretching), 1729 (C=O stretching), 1617 ( $\mathrm{C}=\mathrm{C}$ stretching of aromatic ring). ${ }^{1} \mathrm{H} \mathrm{NMR}\left(300 \mathrm{MHz}, \mathrm{CD}_{3} \mathrm{OD}\right.$

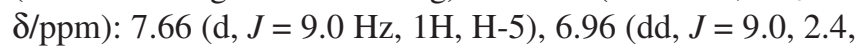
1H, H-6), 6.90 (d, J =2.4 Hz, 1H, H-8), 6.15 (s, 1H, H-3), 6.11-6.02 (m, 1H, H-2), 5.44 (dd, $\left.J=1.5,17.1 \mathrm{~Hz}, \mathrm{H}_{\mathrm{b}}-3\right), 5.27$ (dd , $\left.J=1.5,10.5 \mathrm{~Hz}, \mathrm{H}_{\mathrm{a}}-3\right), 4.66$ (d, $\left.J=5.4 \mathrm{~Hz}, \mathrm{H}-1\right), 2.43$ (s, $\left.3 \mathrm{H}, \mathrm{CH}_{3}-4\right)$. EI-MS: m/z 216[M] $]^{+}, 175\left[\mathrm{C}_{10} \mathrm{H}_{7} \mathrm{O}_{3}\right]^{+}, 159$ $\left[\mathrm{C}_{10} \mathrm{H}_{7} \mathrm{O}_{2}\right]^{+}, 144\left[\mathrm{C}_{9} \mathrm{H}_{4} \mathrm{O}_{2}\right]^{+}, 108\left[\mathrm{C}_{6} \mathrm{H}_{4} \mathrm{O}_{2}\right]^{+}, 51\left[\mathrm{C}_{4} \mathrm{H}_{3}\right]^{+}$.

7-(2-Phenylethoxy)-4-methyl-1-benzopyran-2-one (3e): Buff amorphous powder; yield $64 \%$; m.p. $96-98{ }^{\circ} \mathrm{C}$; m.f.: $\mathrm{C}_{18} \mathrm{H}_{16} \mathrm{O}_{3}$; m.w. 280; IR (KBr, $v_{\max }, \mathrm{cm}^{-1}$ ): 3510 (O-H stretching), 3418 (C-H aromatic stretching), 1723 (C=O stretching), 1613 $\left(\mathrm{C}=\mathrm{C}\right.$ stretching of aromatic ring). ${ }^{1} \mathrm{H} \mathrm{NMR}\left(300 \mathrm{MHz}, \mathrm{CD}_{3} \mathrm{OD}\right.$

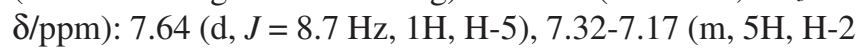
to $\left.6^{\prime}\right), 6.94$ (dd, $\left.J=9.0 \mathrm{~Hz}, 2.4,1 \mathrm{H}, \mathrm{H}-6\right), 6.88(\mathrm{~d}, J=2.4 \mathrm{~Hz}$, $1 \mathrm{H}, \mathrm{H}-8), 6.14$ (s, 1H, H-3), 4.28 (t, $\left.J=6.9 \mathrm{~Hz}, 2 \mathrm{H}, \mathrm{H}-8^{\prime}\right)$, 3.12 (t, $J=6.6 \mathrm{~Hz}, 2 \mathrm{H}, \mathrm{H}-7$ ) $), 2.42$ (s, 3H, $\left.\mathrm{CH}_{3}-4\right)$, EIMS: $\mathrm{m} / \mathrm{z}$ $280[\mathrm{M}]^{+}, 175\left[\mathrm{C}_{10} \mathrm{H}_{7} \mathrm{O}_{3}\right]^{+}, 159\left[\mathrm{C}_{10} \mathrm{H}_{7} \mathrm{O}_{2}\right]^{+}, 144\left[\mathrm{C}_{9} \mathrm{H}_{4} \mathrm{O}_{2}\right]^{+}, 108$ $\left[\mathrm{C}_{6} \mathrm{H}_{4} \mathrm{O}_{2}\right]^{+}, 91\left[\mathrm{C}_{7} \mathrm{H}_{7}\right]^{+}, 51\left[\mathrm{C}_{4} \mathrm{H}_{3}\right]^{+}$.

7-(2-Chlorobenzyloxy)-4-methyl-1-benzopyran-2-one (3f): Creamy white amorphous powder; yield $70 \%$; m.p. 135$137^{\circ} \mathrm{C}$; m.f.: $\mathrm{C}_{17} \mathrm{H}_{13} \mathrm{O}_{3} \mathrm{Cl}$; m.w. 300; IR (KBr, $\left.\nu_{\max }, \mathrm{cm}^{-1}\right)$ : 3515 (O-H stretching), 3416 (C-H aromatic stretching), 1719 (C=O stretching), 1611 ( $\mathrm{C}=\mathrm{C}$ stretching of aromatic ring). ${ }^{1} \mathrm{H} \mathrm{NMR}$ (300 MHz, $\left.\mathrm{CD}_{3} \mathrm{OD} \delta / \mathrm{ppm}\right): 7.72$ (d, $\left.J=8.7 \mathrm{~Hz}, 1 \mathrm{H}, \mathrm{H}-5\right)$, 7.58-7.32 (m, 4H, H-3' to 6) $) 7.05$ (dd, $J=8.7,2.7 \mathrm{~Hz}, 1 \mathrm{H}, \mathrm{H}-$ 6), 6.98 (d, $J=2.4 \mathrm{~Hz}, 1 \mathrm{H}, \mathrm{H}-8), 6.17$ (s, 1H, H-3), 5.27 (s, 
2H, H-7'), 2.44 (s, 3H, $\left.\mathrm{CH}_{3}-4\right)$, EIMS: m/z $300[\mathrm{M}]^{+}, 175$ $\left[\mathrm{C}_{10} \mathrm{H}_{7} \mathrm{O}_{3}\right]^{+}, 159\left[\mathrm{C}_{10} \mathrm{H}_{7} \mathrm{O}_{2}\right]^{+}, 144\left[\mathrm{C}_{9} \mathrm{H}_{4} \mathrm{O}_{2}\right]^{+}, 125\left[\mathrm{C}_{7} \mathrm{H}_{6} \mathrm{Cl}\right]^{+}, 108$ $\left[\mathrm{C}_{6} \mathrm{H}_{4} \mathrm{O}_{2}\right]^{+}, 51\left[\mathrm{C}_{4} \mathrm{H}_{3}\right]^{+}$.

7-(4-Bromobenzyloxy)-4-methyl-1-benzopyran-2-one (3g): Light yellow amorphous powder; yield $72 \%$; m.p. 128$132{ }^{\circ} \mathrm{C}$; m.f.: $\mathrm{C}_{17} \mathrm{H}_{13} \mathrm{O}_{3} \mathrm{Br}$; m.w. 345; IR (KBr, $\left.v_{\max }, \mathrm{cm}^{-1}\right): 3523$ (O-H stretching), 3412 (C-H aromatic stretching), $1725(\mathrm{C}=\mathrm{O}$ stretching), $1616(\mathrm{C}=\mathrm{C}$ stretching of aromatic ring $) .{ }^{1} \mathrm{H}$ NMR (300 MHz, $\mathrm{CD}_{3} \mathrm{OD} \delta / \mathrm{ppm}$ ): 7.70 (d, $\left.J=9.0 \mathrm{~Hz}, 1 \mathrm{H}, \mathrm{H}-5\right)$, 7.55 (d, $J=8.4 \mathrm{~Hz}, 2 \mathrm{H}, \mathrm{H}-3$ ', H-5'), 7.37 (d, $J=8.4 \mathrm{~Hz}, 2 \mathrm{H}$, H-2', H-6'), 7.03 (dd, $J=8.72 .4$ Hz, 1H, H-6), 6.97 (d, $J=2.4$ Hz, 1H, H-8), 6.16 (s, 1H, H-3), 5.15 (s, 2H, H-7), 2.44 (s, $\left.3 \mathrm{H}, \mathrm{CH}_{3}-4\right)$, EI-MS: $m / z 345[\mathrm{M}]^{+}, 175\left[\mathrm{C}_{10} \mathrm{H}_{7} \mathrm{O}_{3}\right]^{+}, 170$ $\left[\mathrm{C}_{7} \mathrm{H}_{6} \mathrm{Br}\right]^{+}, 159\left[\mathrm{C}_{10} \mathrm{H}_{7} \mathrm{O}_{2}\right]^{+}, 144\left[\mathrm{C}_{9} \mathrm{H}_{4} \mathrm{O}_{2}\right]^{+}, 108\left[\mathrm{C}_{6} \mathrm{H}_{4} \mathrm{O}_{2}\right]^{+}, 51\left[\mathrm{C}_{4} \mathrm{H}_{3}\right]^{+}$.

7-(4-Flurobenzyloxy)-4-methyl-1-benzopyran-2-one (3h): Creamy white amorphous powder; yield $85 \%$; m.p. 110 $112{ }^{\circ} \mathrm{C}$; m.f.: $\mathrm{C}_{17} \mathrm{H}_{13} \mathrm{O}_{3} \mathrm{~F}$; m.w. 284; IR (KBr, $\left.\mathrm{v}_{\max }, \mathrm{cm}^{-1}\right): 3511$ (O-H stretching), 3417 (C-H aromatic stretching), 1721 (C=O stretching), 1617 ( $\mathrm{C}=\mathrm{C}$ stretching of aromatic ring). ${ }^{1} \mathrm{H}$ NMR (300 MHz, $\left.\mathrm{CD}_{3} \mathrm{OD} \delta / \mathrm{ppm}\right): 7.67$ (d, $\left.J=8.7 \mathrm{~Hz}, 1 \mathrm{H}, \mathrm{H}-5\right)$, 7.65 (d, $J=8.4 \mathrm{~Hz}, 2 \mathrm{H}, \mathrm{H}-3$ ', H-5 ), 7.13 (d, $J=8.4 \mathrm{~Hz}, 2 \mathrm{H}, \mathrm{H}-$ $2^{\prime}$ and $\mathrm{H}^{-6}$ '), 7.01 (dd, $\left.J=8.7,2.7 \mathrm{~Hz}, 1 \mathrm{H}, \mathrm{H}-6\right), 6.95(\mathrm{~d}, J=$ $2.4 \mathrm{~Hz}, 1 \mathrm{H}, \mathrm{H}-8), 6.13$ (d, J = 0.9 Hz, 1H, H-3), 5.17 (s, 2H, H-7'), $2.42\left(\mathrm{~s}, 3 \mathrm{H}, \mathrm{CH}_{3}-4\right)$, EI-MS: $m / z 284[\mathrm{M}]^{+}, 175$ $\left[\mathrm{C}_{10} \mathrm{H}_{7} \mathrm{O}_{3}\right]^{+}, 159\left[\mathrm{C}_{10} \mathrm{H}_{7} \mathrm{O}_{2}\right]^{+}, 144\left[\mathrm{C}_{9} \mathrm{H}_{4} \mathrm{O}_{2}\right]^{+}, 109\left[\mathrm{C}_{7} \mathrm{H}_{6} \mathrm{~F}\right]^{+}, 108$ $\left[\mathrm{C}_{6} \mathrm{H}_{4} \mathrm{O}_{2}\right]^{+}, 51\left[\mathrm{C}_{4} \mathrm{H}_{3}\right]^{+}$.

7-(4-Chlorobenzyloxy)-4-methyl-1-benzopyran-2-one (3i): White amorphous powder; yield $60 \%$; m.p. $178-180{ }^{\circ} \mathrm{C}$; m.f.: $\mathrm{C}_{17} \mathrm{H}_{13} \mathrm{O}_{3} \mathrm{Cl}$; m.w. 300; IR (KBr, $\left.v_{\max }, \mathrm{cm}^{-1}\right)$ : $3519(\mathrm{O}-\mathrm{H}$ stretching), 3423 (C-H aromatic stretching), 1721 ( $\mathrm{C}=\mathrm{O}$ stretching), 1619 ( $\mathrm{C}=\mathrm{C}$ stretching of aromatic ring). ${ }^{1} \mathrm{H}-\mathrm{NMR}$ (300 MHz, $\mathrm{CD}_{3} \mathrm{OD} \delta / \mathrm{ppm}$ ): 7.67 (d, $\left.J=9.0 \mathrm{~Hz}, 1 \mathrm{H}, \mathrm{H}-5\right)$, $7.43\left(\mathrm{~d}, J=8.4 \mathrm{~Hz}, 2 \mathrm{H}, \mathrm{H}-2^{\prime}\right.$ and H-6'), 7.37 (d, $J=8.4 \mathrm{~Hz}$, 2H, H-3' and H-5'), 7.03 (dd, $J=8.7,2.7 \mathrm{~Hz}, 2.7,1 \mathrm{H}, \mathrm{H}-6$ ), 6.97 (d, $J=2.4 \mathrm{~Hz}, 1 \mathrm{H}, \mathrm{H}-8), 6.15$ (s, 1H, H-3), 5.17 (s, 2H, H-7'), 2.43 (s, 3H, $\left.\mathrm{CH}_{3}-4\right)$, EIMS: $m / z, 300[\mathrm{M}]^{+}, 175$ $\left[\mathrm{C}_{10} \mathrm{H}_{7} \mathrm{O}_{3}\right]^{+}, 159\left[\mathrm{C}_{10} \mathrm{H}_{7} \mathrm{O}_{2}\right]^{+}, 144\left[\mathrm{C}_{9} \mathrm{H}_{4} \mathrm{O}_{2}\right]^{+}, 125\left[\mathrm{C}_{7} \mathrm{H}_{6} \mathrm{Cl}\right]^{+}, 108$ $\left[\mathrm{C}_{6} \mathrm{H}_{4} \mathrm{O}_{2}\right]^{+}, 51\left[\mathrm{C}_{4} \mathrm{H}_{3}\right]^{+}$

4-Methyl-2-oxo-1-benzopyran-7-yl-acetate (3j): White amorphous powder; yield $88 \%$; m.p. $128-130{ }^{\circ} \mathrm{C}$; m.f.: $\mathrm{C}_{12} \mathrm{H}_{10} \mathrm{O}_{4}$; m.w. 218; IR (KBr, $\left.v_{\max }, \mathrm{cm}^{-1}\right)$ : 3534 (O-H stretching), 3412 (C-H aromatic stretching), 1715, 1729 (C=O stretching), $1627\left(\mathrm{C}=\mathrm{C}\right.$ stretching of aromatic ring). ${ }^{1} \mathrm{H} \mathrm{NMR}(300 \mathrm{MHz}$, $\left.\mathrm{CD}_{3} \mathrm{OD} \delta / \mathrm{ppm}\right): 7.69$ (d, $\left.J=8.4 \mathrm{~Hz}, 1 \mathrm{H}, \mathrm{H}-5\right), 7.11$ (dd, $J=$ $8.4 \mathrm{~Hz}, 2.1,1 \mathrm{H}, \mathrm{H}-6), 6.32$ (d, $J=1.2 \mathrm{~Hz}, 1 \mathrm{H}, \mathrm{H}-8), 6.1$ (s, $1 \mathrm{H}, \mathrm{H}-3), 2.4$ (s, 3H, $\left.\mathrm{CH}_{3}-4\right), 2.3$ (s, 3H, $\left.\mathrm{CH}_{3} \mathrm{COO}\right)$. EIMS: $\mathrm{m} / \mathrm{z}$ $218[\mathrm{M}]^{+}, 175\left[\mathrm{C}_{10} \mathrm{H}_{7} \mathrm{O}_{3}\right]^{+}, 159\left[\mathrm{C}_{10} \mathrm{H}_{7} \mathrm{O}_{2}\right]^{+}, 144\left[\mathrm{C}_{9} \mathrm{H}_{4} \mathrm{O}_{2}\right]^{+}, 108$ $\left[\mathrm{C}_{6} \mathrm{H}_{4} \mathrm{O}_{2}\right]^{+}, 51\left[\mathrm{C}_{4} \mathrm{H}_{3}\right]^{+}, 43\left[\mathrm{C}_{2} \mathrm{H}_{3} \mathrm{O}\right]^{+}$.

4-Methyl-2-oxo-1-benzopyran-7-yl-benzoate (3k): Creamy white amorphous powder; yield $78 \%$; m.p. 136$138{ }^{\circ} \mathrm{C}$; m.f.: $\mathrm{C}_{17} \mathrm{H}_{12} \mathrm{O}_{4}$; m.w. 280; IR (KBr, $\left.v_{\max }, \mathrm{cm}^{-1}\right): 3531$ (O-H stretching), 3410 (C-H aromatic stretching), 1718, 1734 $\mathrm{cm}^{-1}$ (C=O stretching), 1623 ( $\mathrm{C}=\mathrm{C}$ stretching of aromatic ring). ${ }^{1} \mathrm{H}$ NMR (300 MHz, $\mathrm{CD}_{3} \mathrm{OD} \delta / \mathrm{ppm}$ ): 8.20-7.84 (m, 5H, H-2' to H-6'), 7.80 (d, $J=9.0 \mathrm{~Hz}, 1 \mathrm{H}, \mathrm{H}-5), 7.30$ (dd, $J=8.7,2.1$ $\mathrm{Hz}, 1 \mathrm{H}, \mathrm{H}-6), 6.90$ (d, J =2.4 Hz, 1H, H-8), 6.30 (s, 1H, H-3), 2.50 (s, 3H, $\left.\mathrm{CH}_{3}-4\right)$, EIMS: m/z $280[\mathrm{M}]^{+}, 175\left[\mathrm{C}_{10} \mathrm{H}_{7} \mathrm{O}_{3}\right]^{+}$, $159\left[\mathrm{C}_{10} \mathrm{H}_{7} \mathrm{O}_{2}\right]^{+}, 144\left[\mathrm{C}_{9} \mathrm{H}_{4} \mathrm{O}_{2}\right]^{+}, 108\left[\mathrm{C}_{6} \mathrm{H}_{4} \mathrm{O}_{2}\right]^{+}, 105\left[\mathrm{C}_{7} \mathrm{H}_{5} \mathrm{O}\right]^{+}$, $51\left[\mathrm{C}_{4} \mathrm{H}_{3}\right]^{+}$.
O-(4-Methyl-2-oxo-1-benzopyran-7-yl)-O-phenylcarbonate (3I): Oily liquid; yield $54 \%$; m.f.: $\mathrm{C}_{17} \mathrm{H}_{12} \mathrm{O}_{5}$; m.w. 296; IR (KBr, $\left.v_{\max }, \mathrm{cm}^{-1}\right)$ : 3529 (O-H stretching), $3409 \mathrm{~cm}(\mathrm{C}-\mathrm{H}$ aromatic stretching), 1706, $1727(\mathrm{C}=\mathrm{O}$ stretching), $1621(\mathrm{C}=\mathrm{C}$ stretching of aromatic ring). ${ }^{1} \mathrm{H}$ NMR $\left(300 \mathrm{MHz}, \mathrm{CD}_{3} \mathrm{OD} \delta /\right.$ ppm): 7.40 (d, $J=8.7 \mathrm{~Hz}, 1 \mathrm{H}, \mathrm{H}-5), 7.17$ (d, $J=2.1 \mathrm{~Hz}, 1 \mathrm{H}$, $\mathrm{H}-8), 6.70$ (dd, $J=8.7,2.1 \mathrm{~Hz}, 1 \mathrm{H}, \mathrm{H}-6), 6.75-6.81$ (m, 5H, H-2' to 6'), 6.00 (s, $1 \mathrm{H}, \mathrm{H}-3), 2.30$ (s, 3H, $\left.\mathrm{CH}_{3}-4\right)$. EIMS: $\mathrm{m} / \mathrm{z}$ $296[\mathrm{M}]^{+}, 175\left[\mathrm{C}_{10} \mathrm{H}_{7} \mathrm{O}_{3}\right]^{+}, 159\left[\mathrm{C}_{10} \mathrm{H}_{7} \mathrm{O}_{2}\right]^{+}, 144\left[\mathrm{C}_{9} \mathrm{H}_{4} \mathrm{O}_{2}\right]^{+}, 108$ $\left[\mathrm{C}_{6} \mathrm{H}_{4} \mathrm{O}_{2}\right]^{+}, 93\left[\mathrm{C}_{6} \mathrm{H}_{5} \mathrm{O}\right]^{+}, 51\left[\mathrm{C}_{4} \mathrm{H}_{3}\right]^{+}$.

in vitro $\boldsymbol{\alpha}$-Chymotrypsin assay: The chymotrypsin inhibitory activity of the compounds was performed by the method of Cannell et al. ${ }^{14}$. Chymotrypsin (9 units $/ \mathrm{mL}$ of 50 mM Tris-HCl buffer pH 7.6; Sigma Chemical Co. USA) was pre-incubated with the compounds for $20 \mathrm{~min}$ at $258^{\circ} \mathrm{C} .100$ $\mathrm{mL}$ of substrate solution (1 mg of $\mathrm{N}$-succinyl-phenylalanine$p$-nitroanilide/mL of $50 \mathrm{mM}$ Tris-HCl buffer $\mathrm{pH}$ 7.6) was added to start the enzyme reaction. The absorbance of released $p$-nitroaniline was continuously monitored at $410 \mathrm{~nm}$ until a significant colour change was achieved. The final DMSO concentration in the reaction mixture was $7 \%$. The percentage (\%) inhibition was calculated as follows (ES)/E x 100, where $\mathrm{E}$ is the activity of the enzyme without test compound and $\mathrm{S}$ is the activity of enzyme in the presence of the test compound. The concentrations of test compounds that inhibited the hydrolysis of substrate upto $50 \%\left(\mathrm{IC}_{50}\right)$ were determined by monitoring the effect of various concentrations of these compounds in the assays on the inhibition values. The $\mathrm{IC}_{50}$ value was then designed using the EZ-Fit Enzyme Kinetics program (Perrella Scientific Inc., Amherst, USA).

Statistical analysis: All the measurements were done in triplicate and statistical analysis was performed by Microsoft Excel 2003. Results are presented as mean \pm sem.

\section{RESULTS AND DISCUSSION}

In the undertaken research, heterocyclic $O$-substituted derivatives of coumarin were synthesized. The parent compound 7-hydroxy-4-methyl-1-benzenpyran-2-one (1), was prepared by the coupling of resorcinol (a) with ethylacetoacetate (b) in the presence of sulphuric acid. Further, the reaction of $\mathbf{1}$ with different electrophiles (2a-l) yielded a series of $O$-substituted derivative of 7-hydroxy-4-methyl-1-benzenpyran-2-one (3al) as represented in Scheme-I. Synthesis of all $O$-substituted derivatives 3a-i was performed in DMF ( $N, N$-dimethylformamide) and sodium hydride $(\mathrm{NaH})$ which act as a base. Complete conversion was achieved within 40-120 min by stirring. The products were isolated by adding cold water in the reaction mixture and filtering off the precipitated solid. In some cases, compound was taken out through solvent extraction method by chloroform/ ethyl acetate. The structure of all the prepared compounds were characterized by ${ }^{1} \mathrm{H}$ NMR, IR and mass spectral data as elaborated in experimental section. Parent compound (1) was synthesized as dark yellow powder. The molecular formula $\mathrm{C}_{10} \mathrm{H}_{8} \mathrm{O}_{3}$ was established by molecular ion peak observed at $m / z 296$ in EI-MS and by counting the number of protons in its ${ }^{1} \mathrm{H}-\mathrm{NMR}$ spectrum. The IR spectrum showed absorption peaks at 3550, 3418, 1717 and $1629 \mathrm{~cm}^{-1}$ which were assigned to $\mathrm{O}-\mathrm{H}$ (stretching of hydroxyl group), $\mathrm{C}-\mathrm{H}$ (aromatic stretching), $\mathrm{C}=\mathrm{O}$ (stretching of carbonyl bond) and 


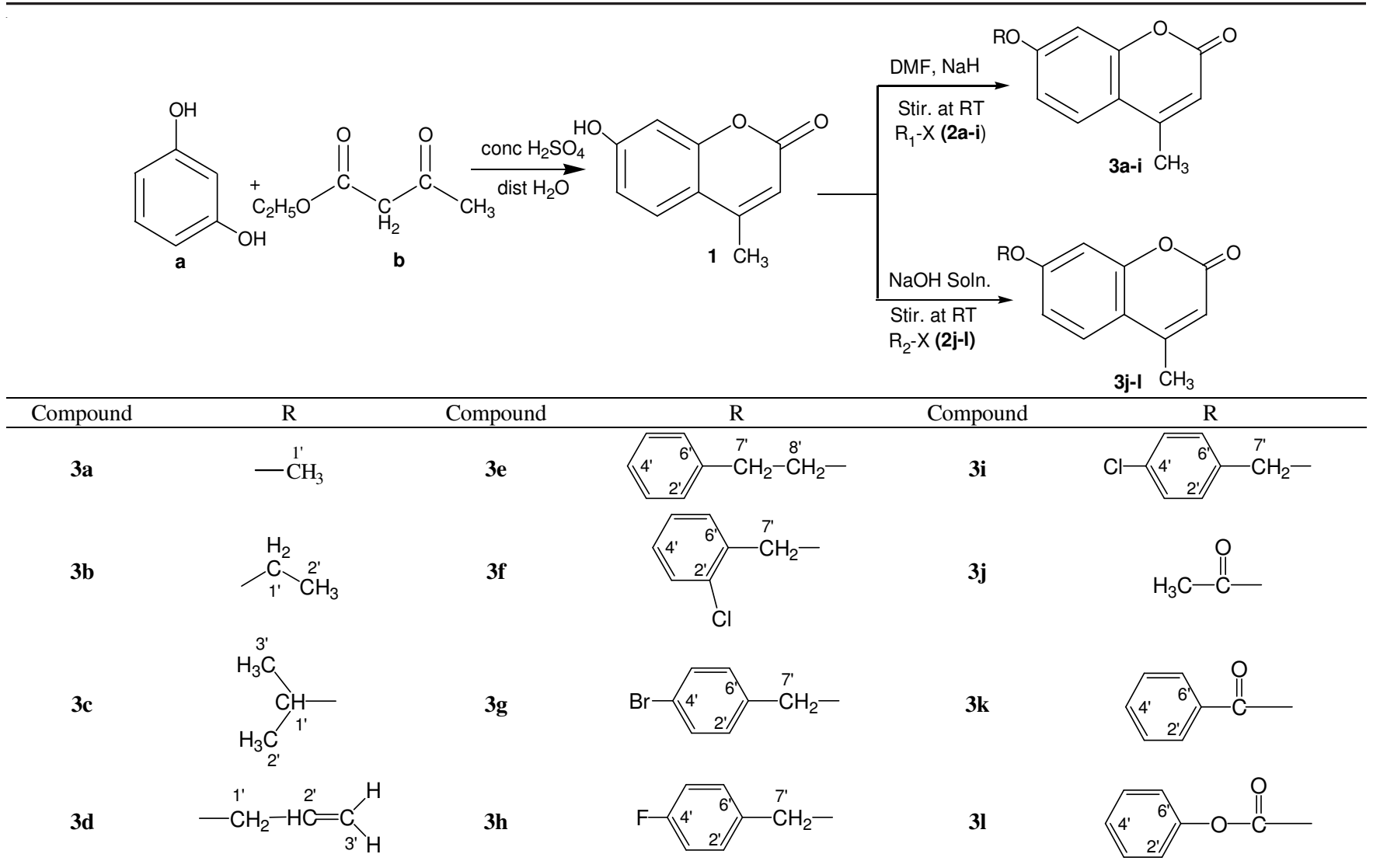

Scheme-1: Outline for the synthesis of $O$-substituted derivatives of 7-hydroxy-4-methyl-1-benzopyarn-2-one

$\mathrm{C}=\mathrm{C}$ (stretching of aromatic ring), respectively. In the ${ }^{1} \mathrm{H}-\mathrm{NMR}$ spectrum, the signals appeared at $\delta 7.58(\mathrm{~d}, J=8.7,1 \mathrm{H}, \mathrm{H}-5)$, 6.82 (dd, $J=8.7,2.4 \mathrm{~Hz}, 1 \mathrm{H}, \mathrm{H}-6), 6.70$ (d, $J=2.4 \mathrm{~Hz}, 1 \mathrm{H}$, $\mathrm{H}-8)$ and $6.09(\mathrm{~d}, J=0.9,1 \mathrm{H}, \mathrm{H}-3)$. In the aliphatic region of the spectrum, signal resonated at $\delta 2.41\left(\mathrm{~d}, J=0.9,3 \mathrm{H}, \mathrm{CH}_{3}-4\right)$ indicated the presence of one methyl group present in the molecule. On the basis of mentioned cumulative evidences, the structure of compound 1 was assigned 7-hydroxy-4-methyl1-benzenpyran-2-one. Mass fragmentation pattern of one of the compound, 7-(4-chlorobenzyloxy)-4-methyl-1-benzopyran2-one (3i) is clearly elaborated in Fig. 1. Similarly, the structures of other 7-O-alkyl/arenyl/acyl derivatives were characterized by IR, ${ }^{1} \mathrm{H}$ NMR and EI-MS data.

All the compounds were screened against $\alpha$-chymotrypsin enzyme and data is given in Table-1. All compounds showed varying degree of enzyme inhibition. It is clearly evident that the compounds 4-methyl-2-oxo-1-benzopyran-7-yl benzoate (3k) and 7-(4-chlorobezyloxy)-4-methyl-1-benzopyran-2-one (3i) were found to be good inhibitors against $a$-chymotrypsin enzyme having $\mathrm{IC}_{50}$ value $58.11 \pm 0.22$ and $115.11 \pm 0.05 \mu \mathrm{mol} / \mathrm{L}$, relative to Chymostatin a reference standard with $\mathrm{IC}_{50}$ value of $8.24 \pm 0.11 \mu$ moles/L, respectively. This indicates that nature of the substituent has great effect on inhibitory activity of these derivatives. These compounds can further be exploited and their derivatives could be synthesized to get closer to $\mathrm{IC}_{50}$ values of the standard, chymostatin. In this way, the compounds could be potential target in the drug discovery and drug development program. Compounds $(\mathbf{3 e}, \mathbf{3 f})$ were found to be the least inhibitor having $\mathrm{IC}_{50}$ value $>400$. Similarly, the compounds $\mathbf{3 a}, \mathbf{3 g}, \mathbf{3} \mathbf{j}$ and $\mathbf{3 l}$ were remained inactive against $\alpha$-chymotrpsin enzyme.

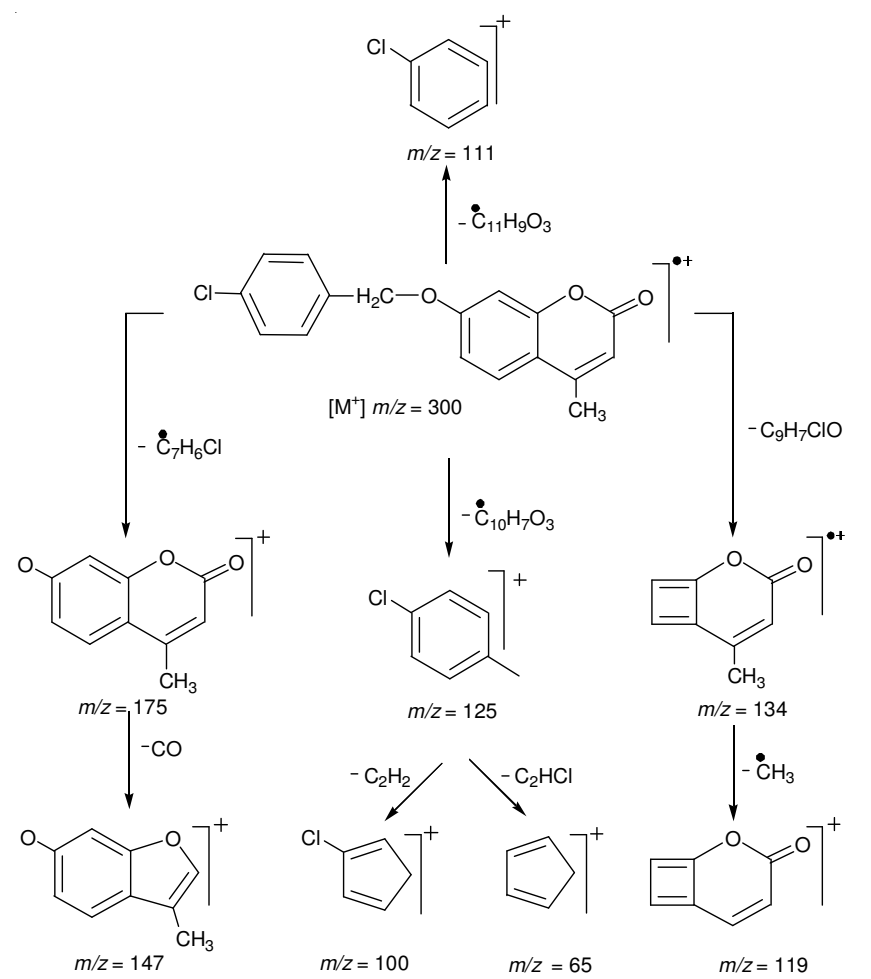

Fig. 1. Mass fragmentation pattern of 7-(4-chlorobezyloxy)-4-methyl-1benzopyran-2-one (3i)

\section{Conclusion}

The projected structures of the synthesized compounds are well supported by spectroscopic data. The synthesized compounds showed varying degree of chymostatin activity. 


\begin{tabular}{|c|c|c|c|}
\hline \multicolumn{4}{|c|}{$\begin{array}{l}\text { TABLE-1 } \\
\text { BIOACTIVITY STUDIES OF } O \text {-SUBSTITUTED DERIVATIVES } \\
\text { OF 7-HYDROXY-4-METHYL-1-BENZOPYARN-2-ONE }\end{array}$} \\
\hline \multirow{2}{*}{ S. No. } & \multicolumn{3}{|c|}{$\alpha$-Chymotrypsin } \\
\hline & Conc. (mM) & Inhibition $(\%)$ & $\mathrm{IC}_{50}(\mu \mathrm{mol})$ \\
\hline 3a & 0.5 & $30.33 \pm 0.11$ & - \\
\hline $\mathbf{3 b}$ & 0.5 & $65.33 \pm 0.11$ & $253.6 \pm 0.01$ \\
\hline $3 \mathbf{c}$ & 0.5 & $72.22 \pm 0.14$ & $239.3 \pm 0.15$ \\
\hline 3d & 0.5 & $64.81 \pm 0.12$ & $229.7 \pm 0.10$ \\
\hline $3 \mathbf{e}$ & 0.5 & $52.45 \pm 0.15$ & $>400$ \\
\hline $3 f$ & 0.5 & $50.68 \pm 0.11$ & $>400$ \\
\hline $3 \mathbf{g}$ & 0.5 & $48.91 \pm 0.16$ & - \\
\hline $3 \mathbf{h}$ & 0.5 & $59.24 \pm 0.19$ & $229.3 \pm 0.11$ \\
\hline $3 \mathbf{i}$ & 0.5 & $65.08 \pm 0.61$ & $115.11 \pm 0.05$ \\
\hline $3 \mathbf{j}$ & 0.5 & $30.43 \pm 0.17$ & - \\
\hline $3 \mathbf{k}$ & 0.5 & $92.93 \pm 0.15$ & $58.11 \pm 0.22$ \\
\hline 31 & 0.5 & $41.58 \pm 0.13$ & - \\
\hline Control & Chymostatin & $93.50 \pm 0.91$ & $8.24 \pm 0.11$ \\
\hline
\end{tabular}

\section{REFERENCES}

1. A.A. Al-Rifai, M.T. Ayoub, A.K. Shakya, K.A. Abu Safieh and M.S. Mubarak, Med. Chem. Res., 21, 468 (2012).

2. F. Maggi, L. Barboni, G. Caprioli, F. Papa, M. Ricciutelli, G. Sagratini and S. Vittori, Fitoterapia, 82, 1215 (2011).

3. M. Raters and R. Matissek, Eur. Food Res. Technol., 227, 637 (2008).

4. S.L. Born, A.M. Api, R.A. Ford, F.R. Lefever and D.R. Hawkins, Food Chem. Toxicol., 41, 247 (2003).

5. D.M. Anderson, S. Shelley, N. Crick and M. Buraglio, J. Clin. Pharmacol., 42, 1358 (2002).

6. M.A. Gouda, M.A. Berghot, E.A. Baz and W.S. Hamama, Med. Chem. Res., 21, 1062 (2011).

7. N.N. Farshori, M.R. Banday, A. Ahmad, A.U. Khan and A. Rauf, Med. Chem. Res., 20, 535 (2011).

8. N. Ingale, V. Maddi, M. Palkar, P. Ronad, S. Mamledesai, A.H.M. Vishwanathswamy and D. Satyanarayana, Med. Chem. Res., 21, 16 (2012).

9. N. Gacche and S.G. Jadhav, J. Exp. Clin. Med., 4, 165 (2012).

10. Y. Shi and C.H. Zhou, Bioorg. Med. Chem. Lett., 21, 956 (2011).

11. I.M. Berquin and B.F. Sloane, Adv. Exp. Med. Biol., 389, 281 (1996).

12. J.L. Imler and J.A. Hoffmann, Curr. Opin. Microbiol., 3, 16 (2000).

13. S. Iwanaga, Curr. Opin. Immunol., 5, 74 (1993).

14. R.J.P. Cannell, S.J. Kellam, A.M. Owsianka and J.M. Walker, Planta Med., 54, 10 (1988). 